\title{
13. THE POLARIZATION OF RADIATION FROM WHITE DWARFS
}

\author{
J. R. P. ANGEL \\ Columbia Astrophysics Laboratory, Columbia University, New York, N.Y., U.S.A. \\ and \\ J. D. LANDSTREET \\ University of Western Ontario, London, Ont., Canada
}

During the course of two observational programs to search for magnetic fields in white dwarfs (Angel and Landstreet, 1970a; Kemp, 1970), it was discovered by Kemp and Swedlund (Kemp et al., 1970b) that the continuum optical radiation from the $\lambda 4135$ type white dwarf $\mathrm{Grw}+70^{\circ} 8247$ is circularly polarized. This circular polarization was measured in a broad band from about 4000 to $7000 \AA$ a number of times; in this band the circular polarization does not appear to vary from its mean value of $3.29 \%$ with an amplitude of more than about $0.1 \%$ (or $3 \%$ of the measured mean polarization) on any time scale from 24 seconds to 2 weeks (Angel and Landstreet, 1970b).

The circular polarization has been measured as a function of wavelength with broad-band filters (Angel and Landstreet, 1970b; Gehrels, 1970). It is found that the circular polarization rises sharply from about $0.75 \%$ at $3300 \AA$ to a maximum of $3.7 \%$ at $4100 \AA$ and then decreases smoothly to about $1.3 \%$ at $9400 \AA$. Further in the infrared, Kemp and Swedlund (1970) have measured the circular polarization to be 8.5 and $15 \%$ at 1.15 and $1.25 \mu$ respectively.

It has also been discovered that the light from Grw $+70^{\circ} 8247$ is linearly polarized, and the linear polarization has been measured as a function of wavelength (Angel and Landstreet, 1970b; Gehrels, 1970). The polarization rises from $2.1 \%$ at $3300 \AA$ to a maximum of $3.7 \%$ at $3800 \AA$, decreases smoothly to approximately zero at $6400 \AA$, and then rises to about $2.8 \%$ at $9400 \AA$. Blueward of $6400 \AA$ the position angle of the linear polarization is about $20^{\circ}$, while at the two measured red points at $8200 \AA$ and $9400 \AA$ it is respectively $101^{\circ}$ and $148^{\circ}$.

A search for circular polarization in 6 DC stars, one DA, and the peculiar DB star HZ29 has not resulted in the discovery of any other circularly polarized white dwarfs (Angel and Landstreet, 1970b). The probable errors have been in the neighborhood of $0.1 \%$.

It has been argued by Kemp (1970) and shown in laboratory experiments (Kemp et al., 1970a) that electronic radiation from a heated radiator in a strong magnetic field is circularly polarized. It is thought that the continuum circular polarization of Grw $+70^{\circ} 8247$ may be due to the presence of a magnetic field of the order of $10^{7} \mathrm{G}$. 


\section{References}

Angel, J. R. P. and Landstreet, J. D.: 1970a, Astrophys. J. (Letters) 160, L147.

Angel, J. R. P. and Landstreet, J. D.: 1970b, Astrophys. J. (Letters) 162, L61.

Gehrels, T.: 1970, private communication.

Kemp, J. C.: 1970, Astrophys. J. $162,169$.

Kemp, J. C. and Swedlund, J. B.: 1970, Astrophys. J. (Letters) 162 L67.

Kemp, J. C., Swedlund, J. B., and Evans, B. D.: 1970a, Phys. Rev. Letters 24, 1211.

Kemp, J. C., Swedlund, J. B., Landstreet, J. D., and Angel, J. R. P.: 1970b, Astrophys. J. (Letters) 161, L77. 